\title{
Statistical Properties of the Excited Binomial States
}

\section{Darwish}

Faculty of Education, Suez Canal University at Al-Arish, Egypt.

The excited binomial state of the radiation field has been recently introduced by repeated application of the photon creation operator on binomial states. In this paper the Glauber second order correlation functions, the quasiprobability distribution functions (Winger function and $Q$-function) for such states are examined.

\section{Introduction:}

Extensive efforts have been paid to generate new states other than number (Fock) states $|n\rangle$ or coherent states $|\alpha\rangle$. The Fock state is the eigenstate of the photon number operator $\hat{n}=\hat{a}^{\dagger} \hat{a}$, i.e. $\hat{a}^{\dagger} \hat{\mathrm{a}}|\mathrm{n}\rangle=n|n\rangle$ where $\hat{a}^{\dagger}$ and $\hat{a}$ are boson creation and annihilation operators respectively. On the other hand, the coherent state is the eigenstate of the annihilation operator $\hat{a}$ i.e. $\hat{a}|\alpha\rangle=\alpha|\alpha\rangle$; besides the value of its normalized second-order correlation function $g^{(2)}(0)$ is unity. Recently, there has been a great deal of interest in producing and generating new states in addition to the usual ones (Fock state and coherent state). Some of the excited quantum states were introduced in the processes of the field-atom interaction in a cavity such as the excited coherent states (ECS) [1]. The ECS exhibits some nonclassical properties such as subpoissonnian photon statistics and squeezing in one of the quadratures of the radiation field, etc. Several other excited quantum states, even and odd ECS [2], excited squeezed states [3-5], and excited thermal states [6, 7] have been studied. These excited quantum states can be prepared by conditional measurement on a beam splitter [8]. It would be interesting to states of the radiation field which can be generated by repeated application of the photon creation operator on binomial states (BS) [9-11] and negative binomial states (NBS) [12-15] are termed as the excited binomial states (EBS) and excited negative binomial states (ENBS) respectively. They reduce to Fock states and excited coherent states in certain limits and can be viewed as intermediate states between Fock states and ECS [16]. In what follows we wish to shed some light on the properties of EBS. 
The organization of the paper is as follows: In section 2 we review briefly the EBS. In section 3 the normalized second-order correlation function is studied. Section 4 is devoted to discuss the quasiprobability distribution functions $Q$-function and (Wigner) $W$-function. Finally, discussion of the result and conclusions are drawn in sec. 5 .

\section{The normalized EBS}

We review briefly the EBS as defined in [16]

$$
\begin{aligned}
|k, \eta, M\rangle & =\lambda(k, \eta, M) \hat{a}^{\dagger k}|n+k\rangle, \\
& \left.=\lambda \sum_{n=0}^{M} C_{n}(\eta, M) \sqrt{\frac{(n+k) !}{n !} \mid} n+k\right\rangle
\end{aligned}
$$

where

$$
|\eta, M\rangle=\sum_{n=0}^{M} C_{n}(\eta, M)|n\rangle
$$

with

Here $|\eta, M\rangle$ is the $\mathrm{BS}$ and $\left|C_{n}(\eta, M)\right|^{2}$ is the probability distribution of finding

$$
C_{n}(\eta, M)=\sqrt{\frac{M !}{n !(M-n) !}} \eta^{n}\left(1-|\eta|^{2}\right)^{\frac{M-n}{2}}
$$

$n$ photons in the BS. Here $k, n$ and $M$ are integers, $\eta$ is a real number, $\hat{a}$ and $\hat{a}^{\dagger}$ satisfy the commutation relation $\left[\hat{a}, \hat{a}^{\dagger}\right]=1$, their effects on the Fock state $|n\rangle$ are

$$
\hat{a}|n\rangle=\sqrt{n}|n-1\rangle, \quad \hat{a}^{\dagger}|n\rangle=\sqrt{n+1}|n+1\rangle,
$$

while $\lambda(k, \eta, M)$ is a normalization constant of the EBS. For determining $\lambda(k, \eta, M)$, the expectation value of the operator $\hat{a}^{k} \hat{a}^{\dagger \mathrm{k}}$ on the BS is calculated as follows

$$
\begin{aligned}
B(k, \eta, M) & =\left\langle\eta, M\left|\hat{a}^{k} \hat{a}^{\dagger \mathrm{k}}\right| \eta, M\right\rangle \\
& =M !\left(1-|\eta|^{2}\right)^{M} \sum_{n=0}^{M} \frac{(n+k) !}{n ! n !(M-n) !}\left(\frac{\eta^{2}}{1-\eta^{2}}\right)^{n}
\end{aligned}
$$


The normalization constant is

$$
\lambda(k, \eta, M)=[B(k, \eta, M)]^{-\frac{1}{2}} .
$$

After the state is being introduced, we shall discuss some of its properties in what follows.

\section{Normalized second-order correlation function}

We shall employ the Glauber second-order correlation function to discuss some statistical properties such as sub-Poissonian distribution [17, 18] which is characteristic of nonclassical states. The condition for sub-Poissonian statistics, for any state, is that the variance $\left\langle(\Delta \hat{n})^{2}\right\rangle=\left\langle\hat{n}^{2}\right\rangle-\langle\hat{n}\rangle^{2}$ must be less than the mean photon number $\langle\hat{n}\rangle$. This can be presented by the Glauber (zero time) second-order correlation function of the form

$$
g^{(2)}(0)=\frac{\left\langle\hat{a}^{\dagger 2} \hat{a}^{2}\right\rangle}{\left\langle\hat{a}^{\dagger} \hat{a}\right\rangle^{2}}=1+\frac{\left\langle(\Delta \hat{n})^{2}\right\rangle-\langle\hat{n}\rangle}{\langle\hat{n}\rangle^{2}} .
$$

A light field has a sub-Poissonian distribution (nonclassical effect) if $g^{(2)}(0)<1$, super-Poissonian distribution effect if $g^{(2)}(0)>1$, and Poissonian distribution if $g^{(2)}(0)=1$. Examples for sub-Poissonian, superPoissonian and Poissonian light statistics are Fock, chaotic and coherent states, respectively. Moreover, the generation of sub-Poissonian light has been established in semiconductor laser [19] and in the microwave region using maser operating in the microscopic regime [20].

To discuss $g^{(2)}(0)$ of EBS we should calculate the expectation values of $\hat{n}=\hat{a}^{\dagger} \hat{a}$ and $\hat{n}^{2}=\left(\hat{a}^{\dagger} \hat{a}\right)^{2}$ with respect to the state, which read as follows: -

$$
\begin{aligned}
& \langle\hat{n}\rangle=|\lambda(k, \eta, M)|^{2} \sum_{n=0}^{M} \frac{M !}{n !(M-n) !}(n+k) \frac{(n+k) !}{n !} \eta^{2 n}\left(1-|\eta|^{2}\right)^{(M-n)} \\
& \left\langle\hat{n}^{2}\right\rangle=|\lambda(k, \eta, M)|^{2} \sum_{n=0}^{M} \frac{M !}{n !(M-n) !}(n+k)^{2} \frac{(n+k) !}{n !} \eta^{2 n}\left(1-|\eta|^{2}\right)^{(M-n)}
\end{aligned}
$$


The autocorrelation function $g^{(2)}(0)$ for this state is plotted in fig. (1) for different values of $M, k$. It is apparent that for $\eta \rightarrow 0$ then $g^{(2)}(0)$ goes to $1-\frac{1}{k}$ for the state $|k, \eta, M\rangle,(k \neq 0)$ and as $\eta \rightarrow 1$ then $g^{(2)}(0)$ tends to $1-\frac{1}{M+k}$. This is depicted clearly in the figure for $g^{(2)}(0)$. The state gives partial coherent light because $0<g^{(2)}(0)<1$. The figures are quantitatively different from that for the BS especially near $\eta=0$ where it tends to very large values for the BS, in contrast to the finite value for the EBS.

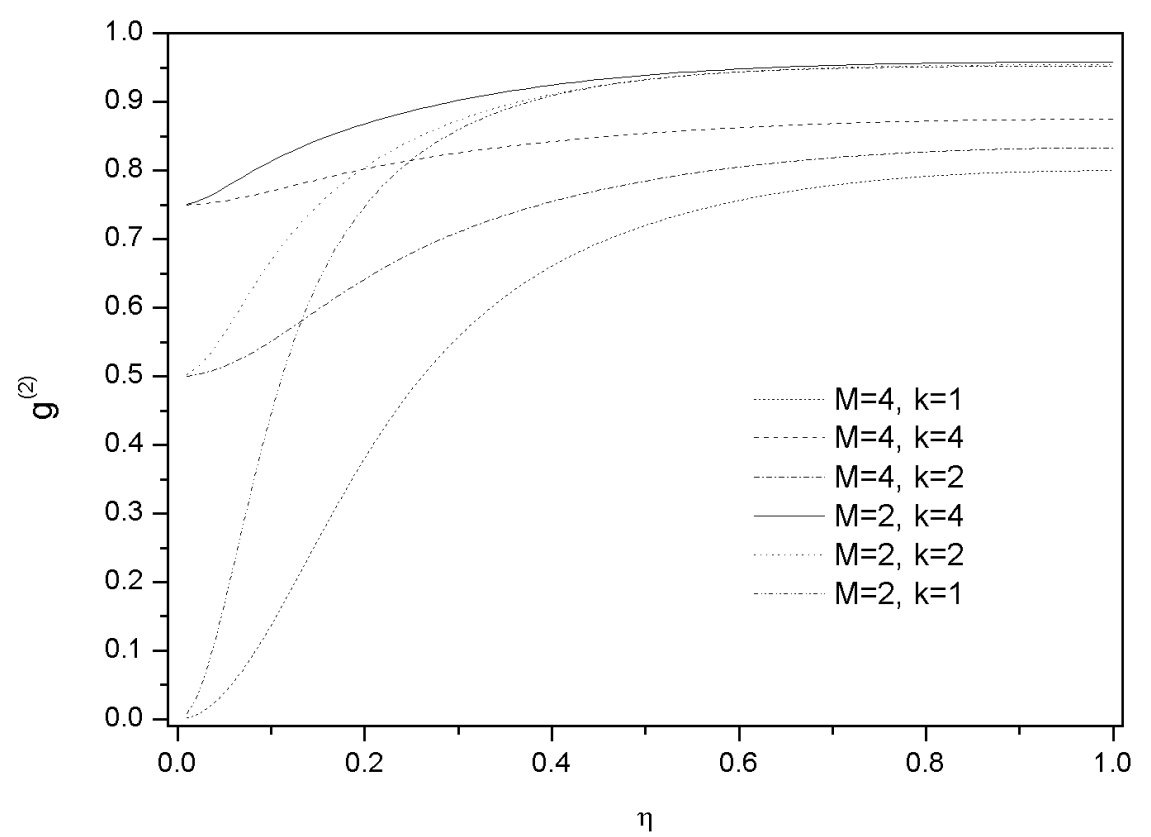

Fig.(1): $g^{(2)}$ parameter as a function of $\eta$ for different values of $M, k$.

\section{Quasi-probability distribution functions}

It has been shown from earlier studies [21-24] that the quasi-probability functions $W$-function, (Husimi) $Q$-function, (Glauber-Sudershan) $P$ function, are important for the statistical description of a microscopic system and provide insight into the nonclassical features of the radiation fields. In this section we shall concentrate on the $Q$-and $W$-functions only. 
The quasi-probability distribution function can be expressed in an integral form through

$$
F(\alpha, s)=\frac{1}{\pi^{2}} \int_{-\infty}^{\infty} C(\beta, s) \exp \left(\alpha \beta^{*}-\alpha^{*} \beta\right) d^{2} \beta
$$

The integration in equation (10) is carried out over the complex $\beta$-plane, where $\beta=|\beta| e^{i \xi}$ and $C(\beta, s)$ is the $s$-ordered generalized characteristic function, which is defined as

$$
C(\beta, s)=\operatorname{Tr}\left[\hat{\rho} \exp \left(\beta \hat{a}^{\dagger}-\beta^{*} \hat{a}\right)\right] \exp \left(\frac{1}{2} s|\beta|^{2}\right)
$$

where $s$ is a parameter that defines the relevant quasi-probability distribution functions. For $s=0$ we obtain the $W$-function, and for $s=-1$ we have the $Q$-function and for $s=1$ the $P$-function is obtained. The function $F(\alpha, s)$ can be represented as a sum over a displaced coherent state $|\alpha, k\rangle$ namely [25]

$$
F(\alpha, s)=\frac{2}{\pi} \sum_{k=0}^{\infty}(-1)^{k} \frac{(1+s)^{2}}{(1-s)^{k+1}}\langle\alpha, k|\hat{\rho}| \alpha, k\rangle
$$

where $\hat{\rho}$ is the density matrix for the EBS given by equation (1). It is well known that the $Q$-function is positive definite at any point of the phase space for any quantum state and can be given in another form as $Q(\alpha)=\pi^{-1}\langle\alpha|\hat{\rho}| \alpha\rangle$. However the $W$-function can take negative values for some states and this is regarded as reflection of the nonclassical effects. By inserting equation (11) into (10), and after performing the integrals and taking $s=0$ or $s=-1$ in equation (12) we obtain expression, for the $W$ - and $Q$ functions. The expression for the $W$-function is:

$$
W(\beta)=\frac{2|\lambda|^{2}}{\pi} e^{-2|\beta|^{2}}\{A+B\},
$$

where $A$ and $B$ are given by 


$$
\begin{aligned}
& A=\sum_{n=0}^{M}(-1)^{n+k} \frac{M !}{(M-n) ! n !} \eta^{2 n}\left(1-|\eta|^{2}\right)^{(M-n)} \frac{(n+k) !}{n !} L_{n+k}\left(4|\beta|^{2}\right) \\
& B=\sum_{m=k+1}^{M} \sum_{n=k}^{m-1}(-1)^{n+k} \sqrt{\frac{n !}{(n+m) !} \times L_{n+k}^{m}\left(4|\beta|^{2}\right) \times 2^{m}\left[\left(\beta^{*}\right)^{m}+(\beta)^{m}\right] \times \rho_{m+n, n}}
\end{aligned}
$$

the Laguerre polynomial $L_{n+k}\left(4|\beta|^{2}\right)$, in equation (14), and the associated Laguerre polynomial $L_{n+k}^{m}\left(4|\beta|^{2}\right)$, in equation (15), are given by:

$$
\begin{aligned}
& L_{n+k}\left(4|\beta|^{2}\right)=\sum_{m=0}^{n+k}(-1)^{m} \frac{(n+k) !\left(4|\beta|^{2}\right)^{m}}{(m !)^{2}(n+k-m) !} \\
& L_{n+k}^{m}\left(4|\beta|^{2}\right)=\sum_{l=0}^{n+k}(-1)^{l} \frac{(n+k+m) !\left(4|\beta|^{2}\right)^{l}}{(l !)(m+l) !(n+k-l) !}
\end{aligned}
$$

and the off-diagonal element of the density operator $\rho_{m+n, n}$ is given by:

$$
\begin{aligned}
\rho_{m+n, n} & =|\lambda|^{2} \sqrt{\frac{n !(n+m) ! !}{(M-n+k) !(M-m-n+k) !}} \\
& \times \frac{M !}{(n-k) !(m+n-k) !} \eta^{(2 n-2 k+m)}\left(1-|\eta|^{2}\right)^{\frac{2 M-2 n+2 k-m}{2}}
\end{aligned}
$$

On the other hand the $Q$-function is given by:

$$
Q(\alpha)=\frac{1}{\pi}|\lambda|^{2} \exp \left(-|\alpha|^{2}\right)|G|^{2}
$$

where

$$
G=\sum_{n=0}^{M} \frac{\alpha^{(n+k)}}{n !} \sqrt{\frac{M !}{(M-n) !} \eta^{n}\left(1-|\eta|^{2}\right)^{\frac{M-n}{2}}}
$$




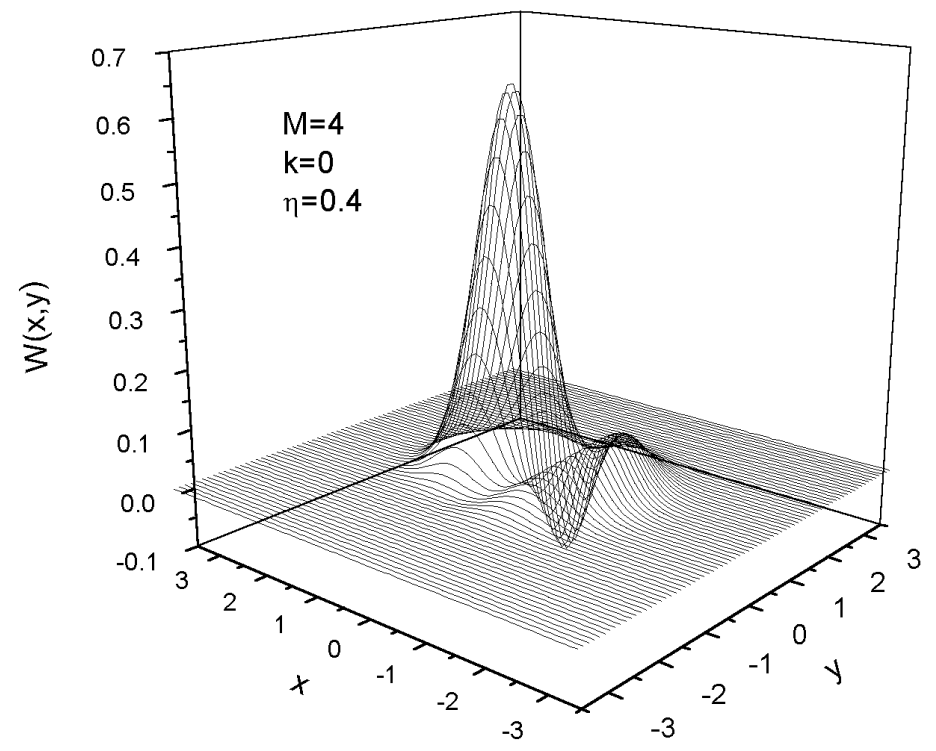

Fig. (2a): W-function as a function of $\alpha$.

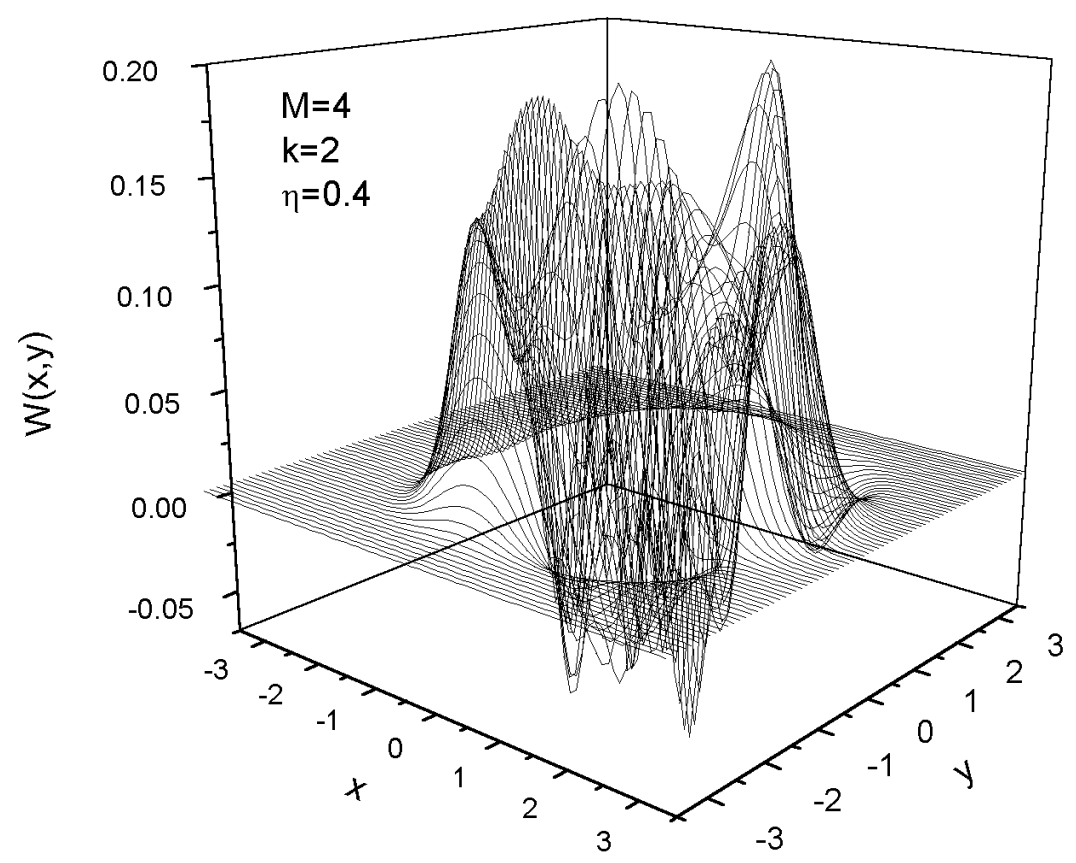

Fig. (2b): W-function as a function of $\alpha$. 


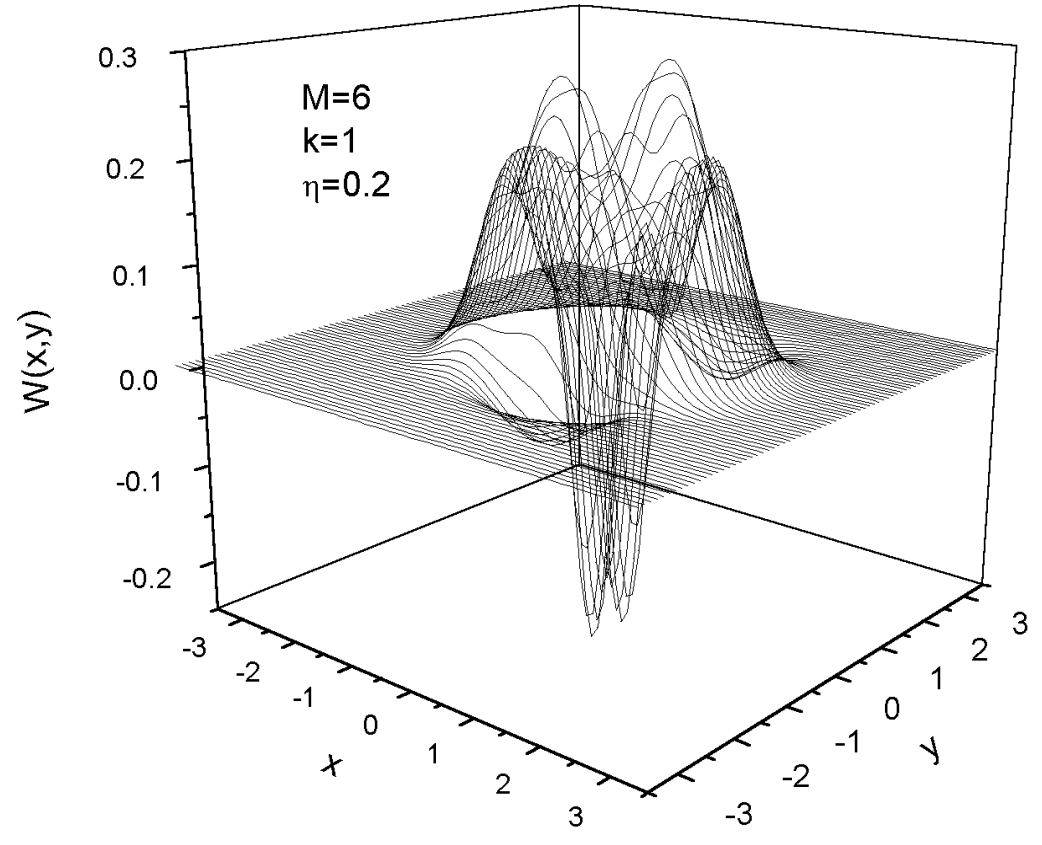

Fig. (2c): W-function as a function of $\alpha$.

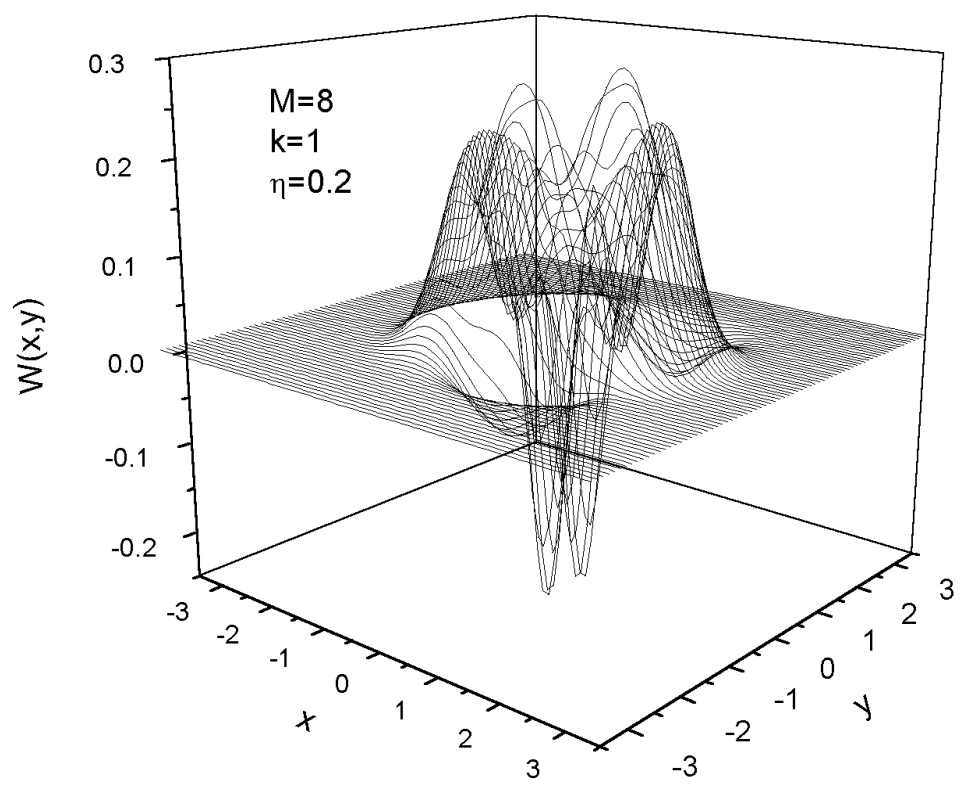

Fig. (2d): W-function as a function of $\alpha$. 
In Figures. (2), one plots the Wigner function for different values of the parameters $M, k$ and $\eta$. The case with $k=0$ which related to the binomial state for $M=4$ and $\eta=0.4$ is plotted in Fig. (2a) where the negative value, meaning a non-classical behaviour is apparent. The figure is asymmetric due to the inclusion of the double summation in the expression (13) for the Wigner function. The general behavior is in agreement with reference [26]. When the excitation is increases to $k=2$, then more wobbles appear. It is to be noted that there is a peak at the origin. The asymmetric behaviour as well as the negativity of the function is observed and more pronounced than the earlier case. When one takes $k=1, M=6$ and $\eta=0.2$, Fig .(2c), one notes that the central peak is inverted downward. This behaviour is noted for all odd $k$, and can be checked from the definition of the Wigner function of equation (13). The increase of $M$ to 8 increases the additional excited states and extends the area of the figure over the $\alpha$-plane. Once again the asymmetry and the appearance of the inverted peak are noted as well as the negative values which the function attain which means non-classical behaviour of the state.

The Husimi $Q$-function is plotted in Fig. (3). The same parameters considered in plotting the Wigner function of Fig. (2) are taken. The plot of the binomial state $|4,0,0.4\rangle$ appears in Fig. (3a). The appearance of an almost Gaussian bell but with little squeezing is one of its axes, shows the deviation from the classical behaviour. As one increases the excitation to $k=2$, Fig.(3b), the asymmetry appears clearly, a crescent like shape is observed for this case. This shape but with the outer ring complete is observed in Fig. (3c, d) when the number $M$ or the factor $\eta$ are increased.

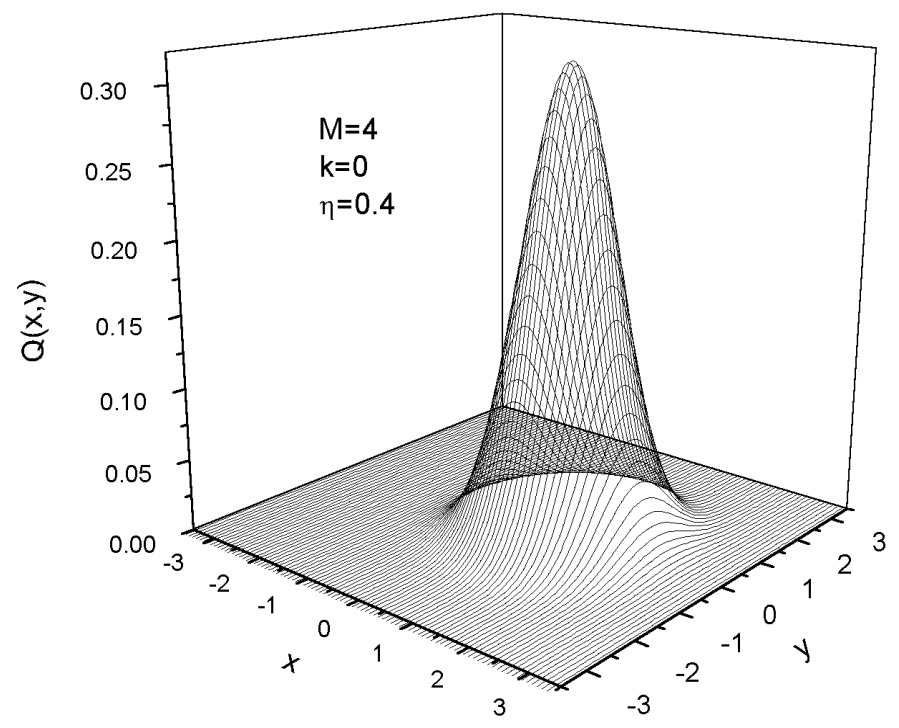

Fig. (3a): Q-function as a function of $\alpha$.

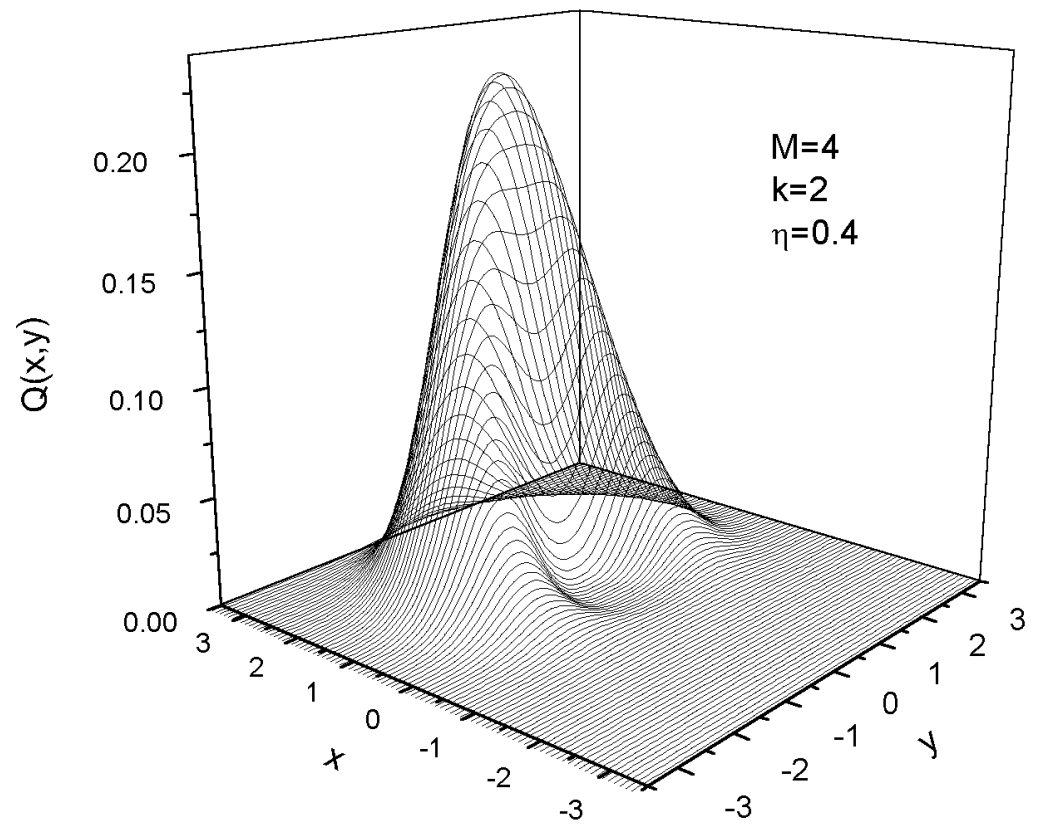




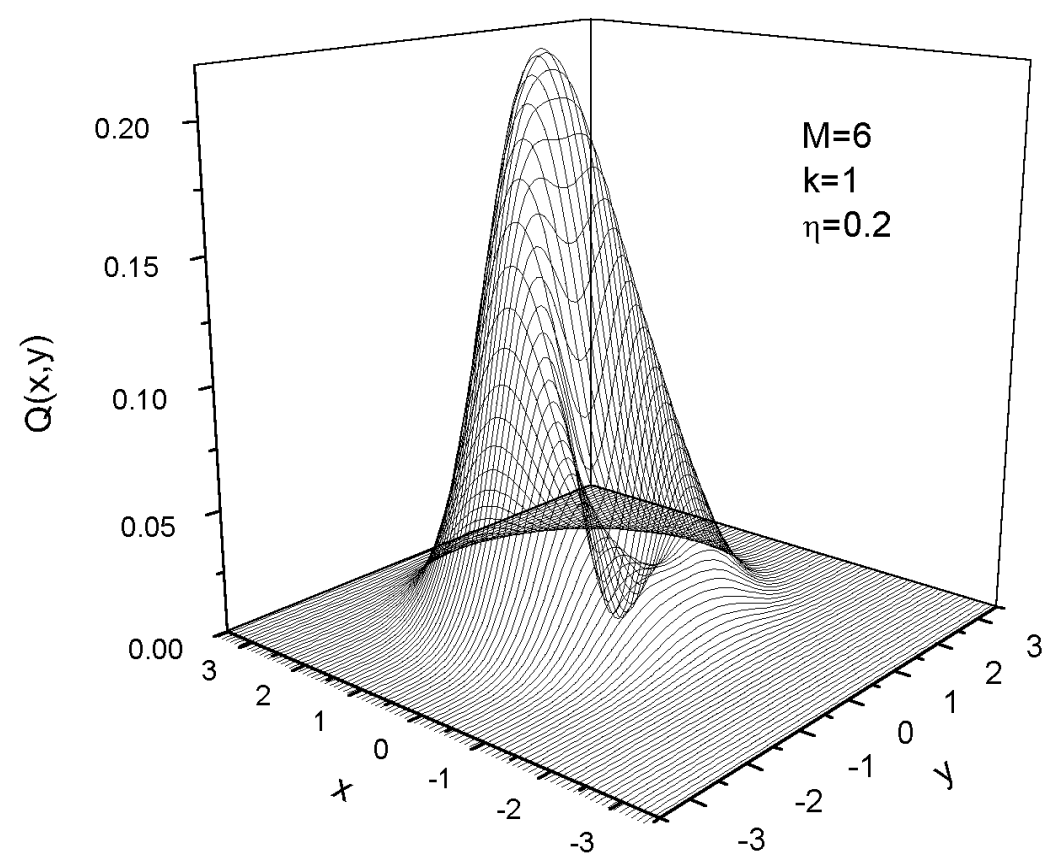

Fig. (3c): Q-function as a function of $\alpha$.

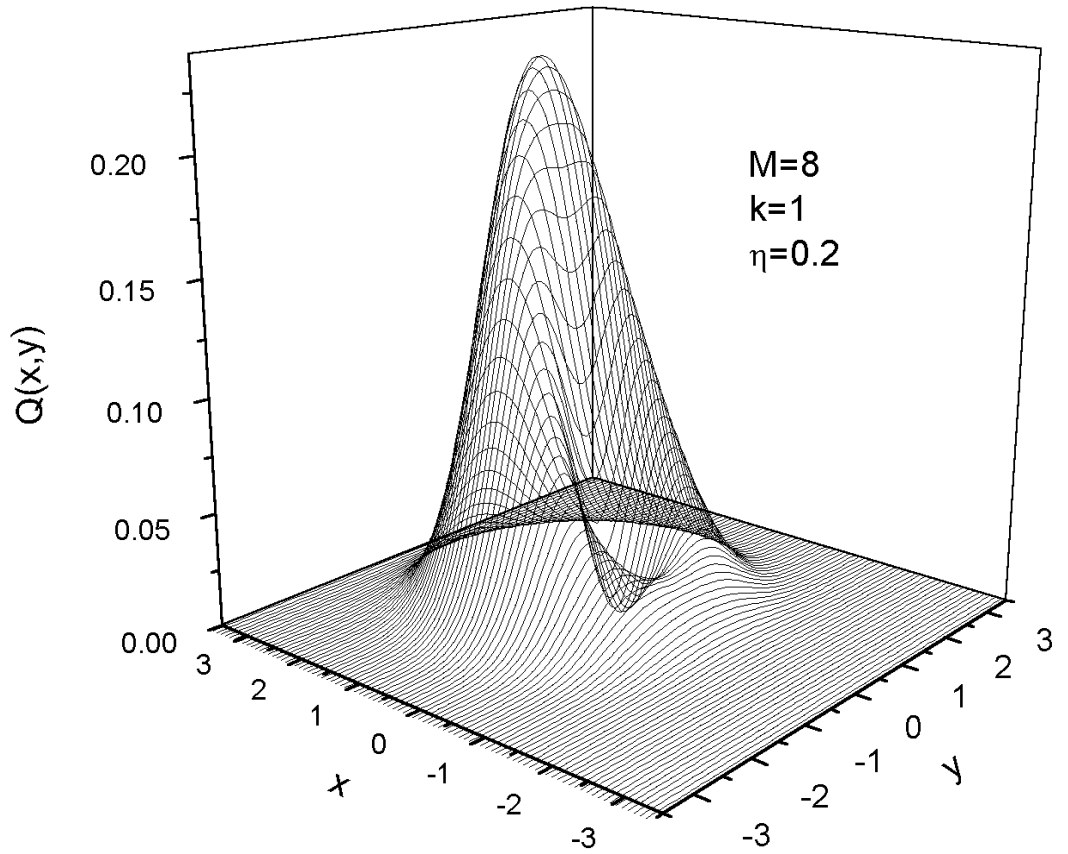




\section{Conclusion:}

In this article we have discussed some statistical properties of the EBS. The second-order autocorrelation function $g^{(2)}(0)$ is investigated.

It is found that as $\eta \rightarrow 0$ the curves differ qualitatively from those characteristics for the BS. This is due to the inclusion of the state $|k\rangle$ as $\eta \rightarrow 0$ for EBS in contrast to the state $|0\rangle$ for the BS. The quasidistribution functions especially $W$ - and $Q$-functions are discussed non-classical effects are exhibited since it is found that the $W$-function assumes negative values for certain range of the domain. Our concentration was on the averaging case, where we found for small value of $|\eta|$ the Wigner function is almost Gaussian. However, the shape of the function is insensitive to change in $M$. In contrast we find that the $Q$-function is insensitive to any change in either $|\eta|$ or $M$.

\section{References:}

1. G. S. Agarwal, and K. Tara, Phys. Rev. A43, 492(1991) 
2. V. V. Dodonov, Ya. A. Korennoy, V. I. Man'ko and Y. A. Moukhin, Quantum Semiclass. Opt. 8, 413(1996)

3. Z. Zhang and H. Fan, Phys. Lett. A165, 14(1992)

4. Zs. Kis, P. Adam and J. Janzky, Phys. Lett. A188, 16(1994)

5. V. I. Man'ko and A. Wunsche, Quantum Semiclass. Opt. 9, 381(1997)

6. G. S. Agarwal and K. Tara, Phys. Rev. A46, 485(1992)

7. G. N. Jones, J. Haight and C. T. Lee, Quantum Semiclass. Opt. 9, 411(1997)

8. M. Dakna, L. Knoll and D. G. Welsh, Optica Comm Unvcations 145 , 309(1998)

9. D. Stoler, B. E. A. Saleh and M. C. Teich, Opt. Acta 32, 345(1985)

10. G. Dattoli, J. Gallardo and A., J. Torre, Opt. Soc. Am. B2, 185(1987)

11. H. C. Fu and R. Sosaki, J. Phys. 24, 483(1996)

12. K. Matsuo, Phys. Rev. A41, 519(1990)

13. A. Joshi, and A.-S.F. Obada, J. Phys. A: Math. Gen. 30, 81(1997)

14. H. C. Fu and R. Sasaki, J. Phys. Soc. Japan 66, 1989(1997)

15. S. M. Barett, J. Mod. Opt. 45, 2201(1998)

16. X. G. Wang and H. C. Fu, Int. J. Th. Phys. 39, 1437(2000)

17. J. Perina, "Quantum statistics of linear and nonlinear optical phenomena", Reidel, Dordrecht, P. 78(1984)

18. M. Hillery, Phys. Rev. A36, 3796(1987)

19. Y. Yamamoto and S. Machida, Phys. Rev. A35, 5114(1987)

20. G. Rempe, F. Schmidt-Kaler and H. Walther, Phys. Rev. Lett. 64, 2783(1990)

21. E. Wigner, Phys. Rev. 40, 749(1932)

22. Z. Wigner Phys. Chem. B19, 203(1932)

23. K. E. Cahill, and R. J. Glauber, Phys. Rev. 177, 1882(1969)

24. M. Hillery, R. F. O'Connell, M. O. Scully and E. P. Winger, Phys. Rep. 106, 121(1984)

25. H. Moya-Cessa and P. L. Knight, Phys. Rev. A48, 2479(1993)

26. A. Vidiella-Barranco and J. A. Roversi, Phys. Rev. A50, 5233(1994) 